\title{
Case - Malakoplakia in a 58-year-old male following living donor renal transplantation
}

Nikhile Mookerji, BSc ${ }^{1}$; Thomas Skinner, MD, FRCSC ${ }^{2}$; Kristen McAlpine, MD $^{2}$; Jeffrey Warren, MD, FRCSC ${ }^{1,2}$

${ }^{1}$ School of Medicine; ${ }^{2}$ Divsion of Urology; University of Ottawa, Ottawa, ON, Canada

Cite as: Can Urol Assoc J 2018 July 24; Epub ahead of print.

http://dx.doi.org/10.5489/cuaj.5282

Published online July 24, 2018

$* * *$

\section{Introduction}

Malakoplakia is a granulomatous disease originally named by Von Hansemann in 1903 following the first description of the condition by Michaelis and Gutmann one year prior. ${ }^{1}$ The name was derived from the macroscopic appearance of 'soft plaques' (which in Greek translates to malakos plakos). ${ }^{1}$ Malakoplakia is a rare pseudotumor that arises in the context of recurrent infections, particularly in the immunocompromised setting. ${ }^{2}$ It is most commonly found in the urinary tract but has been reported in other organs including the gastrointestinal tract, skin, and lungs. ${ }^{3}$ While the exact pathophysiology of the disease state remains unknown, it is thought to involve dysfunctional tissue macrophages termed von Hansemann cells. ${ }^{1}$ Histologically, Michaelis-Gutmann bodies are basophilic cellular inclusions and are pathognomonic for the diagnosis of malakoplakia. ${ }^{2}$

\section{Case report}

A 58-year-old obese male (body mass index $35.3 \mathrm{~kg} / \mathrm{m}^{2}$ ) with end stage renal disease secondary to autosomal dominant polycystic kidney disease received a preemptive living donor renal transplant in March 2014. The patient's early postoperative course was unremarkable with negative urine cultures, stable creatinine (nadir of $70 \mu \mathrm{mol} / \mathrm{L}$ ), and normal voiding. Induction immunosuppression was achieved with basiliximab, cyclosporine, and sirolimus. This was followed by maintenance therapy with tacrolimus (3mg daily), mycophenolate (2g daily), and prednisone (5mg daily). His creatinine remained relatively stable $(70-130 \mu \mathrm{mol} / \mathrm{L})$ postoperatively until May 2014 when it began to rise insidiously. In July, he had two separate allograft biopsies for increasing creatinine. The first biopsy identified Banff type 1B acute cellular rejection and the second, showed type 1A acute cellular rejection. In August 2014 a third biopsy revealed only acute tubular necrosis. The patient's creatinine continued to rise during this time to a new baseline of $170 \mu \mathrm{mol} / \mathrm{L}$. He received thymoglobulin and pulsed steroids to treat the rejection episode. Imaging was performed and revealed a pelvic fluid collection that was drained 
percutaneously. The drain fluid was sent for bacterial culture and grew Enterobacter cloacae and Escherichia coli (E. coli). This was treated with Ciprofloxacin 500mg IV BID for 7 days.

In September, the patient returned to the emergency department with night sweats, chills, increasing abdominal pain, and oliguria. Creatinine had increased to $300 \mu \mathrm{mol} / \mathrm{L}$ and an ultrasound revealed hydronephrosis of the allograft. A computed tomography (CT) scan demonstrated multiple abdominal wall abscesses, which were drained percutaneously. This fluid was also positive for $E$. coli. A nephrostomy tube was inserted to decompress the allograft collecting system and urine culture grew Klebsiella pneumoniae. Initially the patient was treated with intravenous (IV) Piperacillin/Tazobactam, but was transitioned to IV Ertapenem after antibiotic sensitivities returned. A non-contrast CT scan was repeated in October and identified a new, solid peritransplant mass (Figure 1). Given this unexpected finding, a contrast-enhanced CT scan was arranged. This revealed heterogeneous enhancement of this mass with a compressive effect on the allograft renal vein and left external iliac vein. The external iliac, internal iliac, and proximal portion of the common femoral veins all demonstrated radiographic evidence of thrombosis. Decreased perfusion to the upper pole of the transplant kidney was noted in addition to a pulmonary embolism in the right lower lobe pulmonary artery. CT guided biopsy of the solid peritransplant mass was sent for pathologic analysis promptly thereafter (Figure 2).

The patient was started on IV ceftazidime, IV vancomycin and anticoagulation. Mycophenolate and prednisone were stopped and the dose of tacrolimus was reduced to target trough levels of 4-6 $\mu \mathrm{g} / \mathrm{L}$. Intravenous ganciclovir was also initiated for CMV viremia. Despite these efforts, the graft function continued to deteriorate with the serum creatinine reaching 490 $\mu \mathrm{mol} / \mathrm{L}$ by October 31, 2014. The patient ultimately required initiation of hemodialysis. He went on to receive an allograft nephrectomy in January 2017 to facilitate the possibility of a second transplantation.

\section{Discussion}

Malakoplakia is a chronic granulomatous disease that uncommonly arises in renal transplant recipients. ${ }^{3}$ As demonstrated in this case, it is usually found in the context of recurrent infections, and can contribute to loss of graft function. ${ }^{1-4}$ In renal transplant recipients who develop malakoplakia, E. coli is present in $80 \%$ of cases. ${ }^{3,4}$ Other pathogens that have been reported include Klebsiella, Pseudomonas, Enterococcus, Streptococcus, and Staphylococcus. ${ }^{3,6}$

While the pathophysiology of malakoplakia is not completely understood, Abdou et al. (1977) remain one of the only groups to have studied the mechanism of this disease process. The authors propose that malakoplakia results from defects in monocytes that lead to abnormally large lysosomal granules with low levels of cyclic guanosine monophosphate (cGMP). ${ }^{5}$ This is felt to result in poor release of beta-glucuronidase from leukocytes exposed to opsonized microbes causing blunted bactericidal activity. First-line treatment of malakoplakia remains long courses of antibiotics and decreased doses of immunosuppressants. ${ }^{3,4,6}$ Recommended antibiotics 
include fluoroquinolones and trimethoprim-sulfamethoxazole given their ability to work within macrophages. ${ }^{8}$

For surgical residents and staff physicians caring for renal transplant recipients, malakoplakia is a rare finding that may not be high on the initial list of differential diagnoses for post-operative complications. However, in cases where there are multiple abscesses, deteriorating graft function, and a new mass or pseudo tumor identified in or around the graft malakoplakia should be considered. An important component of early diagnosis and treatment is biopsy. This should be considered for any renal mass or tumor found in proximity to an allograft.

Identification of malakoplakia requires special cytologic staining with periodic acidschiff dye and von Kossa staining. On microscopic assessment, Michaelis-Gutmann bodies are pathognomonic for malakoplakia. Malakoplakia is not easily identified with traditional histological stains and may be underreported because it is missed if not tested for. ${ }^{6}$ Additionally, non-contrast CT scans can occasionally identify hyperdense or calcified masses as the first sign of malakoplakia. This occurs because of the abundance of phagolysosomes within von Hansemann cells which contain intact or partially digested bacteria and are encrusted with calcium and iron. ${ }^{1-3,5}$

A 2012 review of the literature, identified a decrease in rate of reported malakoplakia cases in renal transplant patients, after the change in routine immunosuppressive therapy from cyclosporine and azathioprine to tacrolimus and mycophenolate. ${ }^{6}$ Other studies, however, have found that this regimen increases the risk of opportunistic infections which could potentially increase the rates of malakoplakia over time. ${ }^{7}$ The long term effect on malakoplakia rates due to changes in immunosuppressive regimes post-transplant remains unclear.

Finally, the prognosis of malakoplakia depends on many factors including the patient's overall health, type of immunosuppression and the location of involvement. ${ }^{3}$ The case presented was unique due to the encasement and obstruction of both the iliac and renal blood vessels. This

presumably contributed to the thrombosis and eventual graft failure. Ultimately, the patient went on to require an allograft nephrectomy 3 years post-transplant and is currently managed on intermittent hemodialysis. While most cases of malakoplakia are benign and respond to antibiotics, this disease can cause significant morbidity and the need for surgical intervention. $3,4,8,9$

\section{Conclusion}

Malakoplakia is a rare granulomatous condition that can arise in organ transplant recipients. Given the potential for significant morbidity if missed, malakoplakia should be included in the differential diagnosis of renal transplant recipients who present with recurrent urinary tract infections or acute kidney injury with an associated mass in the urinary tract. Treatment should include multi-disciplinary involvement, antibiotics, and reduction in immunosuppressive medications. This condition may ultimately compromise graft function and in severe cases may require allograft nephrectomy. 


\section{References}

1. McClure, J. Malakoplakia. J Pathol 1983;140:275-330.

2. Dobyan, D. C., Truong, L. D., \& Eknoyan, G. Renal malacoplakia reappraised. Am J Kidney Dis 1993;22:243-52.

3. Nieto-Ríos, J. F., Ramírez, I., Zuluaga-Quintero, M., et al. Malakoplakia after kidney transplantation: case report and literature review. Transpl Infect Dis 2017;19:e12731

4. Augusto, J. F., Sayegh, J., Croue, A., et al. Renal transplant malakoplakia: case report and review of the literature. NDT plus 2008;1:340-43.

5. Abdou, N. I., NaPombejara, C., Sagawa, A., et al. Malakoplakia: evidence for monocyte lysosomal abnormality correctable by cholinergic agonist in vitro and in vivo. $N$ Engl $J$ Med 1977;297:1413-19.

6. Leao, C. A., Duarte, M. I. S., Gamba, C., et al. Malakoplakia after renal transplantation in the current era of immunosuppressive therapy: case report and literature review. Transpl Infect Dis 2012;14:E137-41

7. Parasuraman, R., Abouljoud, M., Jacobsen, G., et al. Increasing trend in infection-related death-censored graft failure in renal transplantation. Transplantation 2011;91:94-9.

8. Graves, A. L., Texler, M., Manning, L., et al. Successful treatment of renal allograft and bladder malakoplakia with minimization of immunosuppression and prolonged antibiotic therapy. Nephrology (Carlton) 2014;19:18-1

9. Arnesen, E., Halvorsen, S., Skjørten, F. Malacoplakia in a renal transplant: report of a case studied by light and electron microscopy. Scand J Urol Nephrol 1977;11:93-6.

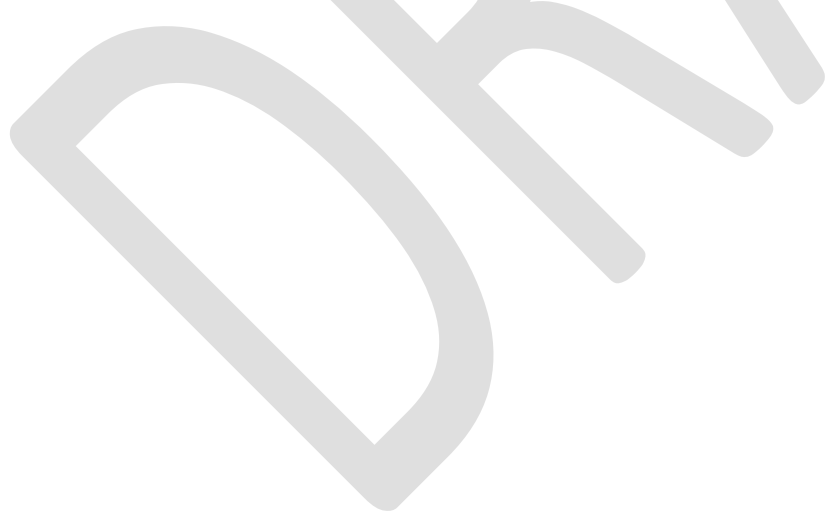




\section{Figures and Tables}

Fig. 1. Heterogeneously enhancing mass adjacent to the hilum of the transplanted kidney (yellow arrow). Anterior component of mass tethered to the left rectus abdominis muscle (green arrow). Left external iliac artery seen being encased within mass (red arrow). External Iliac vein also encased within mass but difficult to demarcate (blue arrow). Nephrostomy tube can be appreciated (purple arrow).
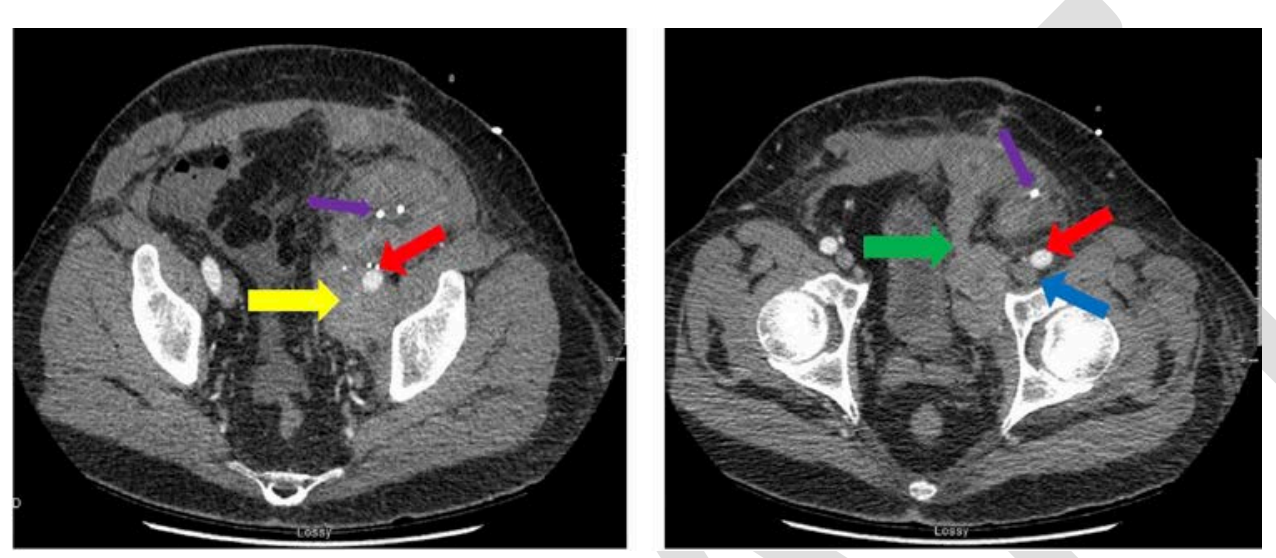

Fig. 2. Histological slides from computed tomography (CT)-guided biopsy of hilar mass. Pathological review revealed numerous histiocytes arranged in sheets. The histiocytes had abundant granular cytoplasm (PAS positive, stain not shown). The nuclei were monomorphic and mitotic figures were absent. Concentrically layered extracellular structures consistent with Michaelis-Gutman bodies (indicated by arrows) were highlighted by Von Kossa stains. The overall findings are in keeping with malakoplakia.

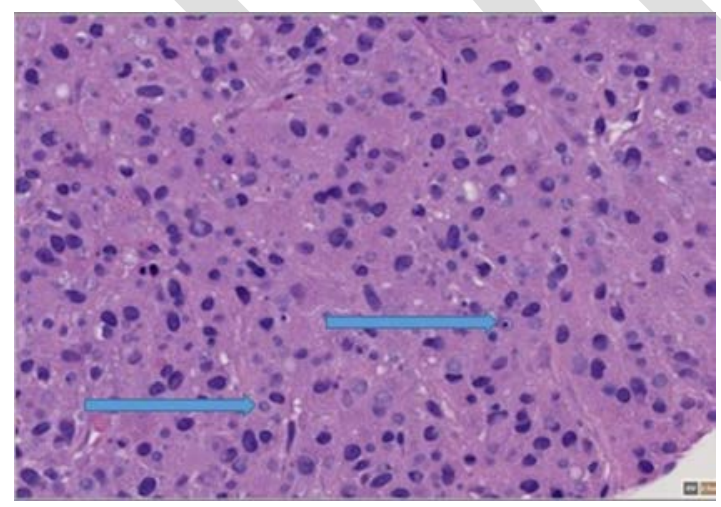

